\title{
Waveguide Propagation in Extended Plates of Variable Thickness
}

\author{
Safarov Ismail Ibragimovich, Akhmedov Maqsud Sharipovich, \\ Boltaev Zafar Ihterovich \\ Bukharian Engineering and Institute of Technology, Bukhara, Republic of Uzbekistan \\ Email: safarov54@mail.ru, maqsud.axmedov.1985@mail.ru
}

Received 6 November 2014; revised 12 December 2014; accepted 29 December 2014

Copyright (C) 2014 by authors and OALib.

This work is licensed under the Creative Commons Attribution International License (CC BY).

http://creativecommons.org/licenses/by/4.0/

c) $\underset{\mathrm{EY}}{\mathrm{B}}$ Open Access

\begin{abstract}
In this paper we construct conjugate spectral problem and the conditions of biorthogonality for distribution in extended plates of variable thickness of the problem considered. It describes the procedure of solving problems and a numerical result is on wave propagation in an infinitely large plate of variable thickness. Viscous properties of the material are taken into account by means of an integral operator Voltaire. Research is conducted in the framework of the spatial theory of visco elastic. The technique is based on the separation of spatial variables and formulates the boundary eigenvalue problem that can be solved by the method of orthogonal pivotal condensation Godunov. Numerical values obtained the real and imaginary parts of the phase velocity depending on the wave numbers. The numerical result coincides with the known data.
\end{abstract}

\section{Keywords}

Biorthogonality, Wave Propagation, Variable Thickness

Subject Areas: Mathematical Analysis, Mechanics

\section{Introduction}

It is known [1] [2] that normal wave deformable layer (Lamb wave) is not orthogonal to the thickness, i.e. the integral of the scalar product of vectors of displacements of two different waves, considered as functions of the coordinate perpendicular to the surface layer, is not zero. They are also not orthogonal conjugate waves that are obtained by considering the dual problem. This fact introduces additional difficulties in solving practical problems [3]-[5]. In this paper we construct conjugate spectral problem and biorthogonality conditions for the problem. 


\section{The Mathematical Formulation of the Problem}

We consider the viscoelastic waveguide in the form of an endless along the axis $x_{1}$ of the variable thickness (Figure 1).

The main relations of the classical theory of plates of variable thickness can receive based on the principle of possible displacements. Variation equation problem of the theory viscose of elasticity in a three-dimensional formulation has the form

$$
\iiint_{V}\left(\sigma_{i j} \delta \varepsilon_{i j}+\rho \ddot{u}_{i} \delta u_{i}\right) \mathrm{d} x_{3} \mathrm{~d} x_{2} \mathrm{~d} x_{1}=0, \quad \ddot{u}_{i}=\frac{\partial^{2} u_{i}}{\partial t^{2}}, \quad(i=1,2,3 ; j=1,2,3)
$$

where $\rho$-density of the material; $u_{i}$-the displacement components; $\sigma_{i j}$ and $\varepsilon_{i j}$-tensor components of stress and strain; $V$ — volume occupied by the body. In accordance with the hypotheses of Kirchhoff-Lava [5]

$$
\sigma_{12}=\sigma_{23}=\sigma_{33}=0, \quad u_{i}=-x_{3} \frac{\partial W}{\partial x_{i}} .
$$

Neglecting in (1) the members of that take account the rotational inertia of the normal to median plane, will have the following variation equality:

$$
\int_{S} \mathrm{~d} s \int_{-\frac{h}{2}}^{\frac{h}{2}}\left(\sigma_{11} \delta \varepsilon_{11}+2 \sigma_{12} \delta \varepsilon_{12}+\sigma_{22} \delta \varepsilon_{22}\right) \mathrm{d} x_{3}+\int_{S} \mathrm{~d} s \int_{-\frac{h}{2}}^{\frac{h}{2}} \rho \frac{\partial^{2} W}{\partial t^{2}} \delta W \mathrm{~d} x_{3}=0
$$

Proceeding from geometric relations and ratios of generalized Hooke law, taking into account of kinematic of hypotheses (2), the expressions for the components strain and stress tensors has the form

$$
\begin{aligned}
& \varepsilon_{i j}=\frac{1}{2}\left(\frac{\partial u_{i}}{\partial x_{j}}+\frac{\partial u_{j}}{\partial x_{i}}\right)-x_{3} \frac{\partial^{2} W}{\partial x_{i} \partial x_{j}}, \quad i, j=1,2 ; \\
& \sigma_{11}=\frac{\tilde{E}}{1-v}\left(\varepsilon_{11}+v \varepsilon_{22}\right) ; \quad \sigma_{22}=\frac{\tilde{E}}{1-v}\left(\varepsilon_{22}+v \varepsilon_{11}\right) ; \quad \sigma_{12}=\frac{\tilde{E}}{1+v} \varepsilon_{12} .
\end{aligned}
$$

where $\tilde{E}$-the modulus of elasticity operator, which is [6],

$$
\tilde{E} \varphi(t)=E_{01}\left[\varphi(t)-\int_{-\infty}^{t} R_{E}(t-\tau) \varphi(t) \mathrm{d} \tau\right]
$$

$\varphi(t)$ - An arbitrary function of time; $R_{E}(t-\tau)$ - Relaxation kernel; $E_{01}$ - Instantaneous modulus of elasticity; $v$-Poisson's ratio, it is suggested that a constant value; take the integral terms in (5) small, then the function $\varphi(t)=\psi(t) \mathrm{e}^{-i \omega_{R} t}$, where $\psi(t)$-is a slowly varying function of time, $\omega_{R}$-be a real constant. Next, using the procedure of freezing [7], we note the relation (2) the approximate form

$$
\bar{E} \varphi=\lambda_{m}\left[1-\Gamma^{C}\left(\omega_{R}\right)-i \Gamma^{S}\left(\omega_{R}\right)\right] \varphi
$$

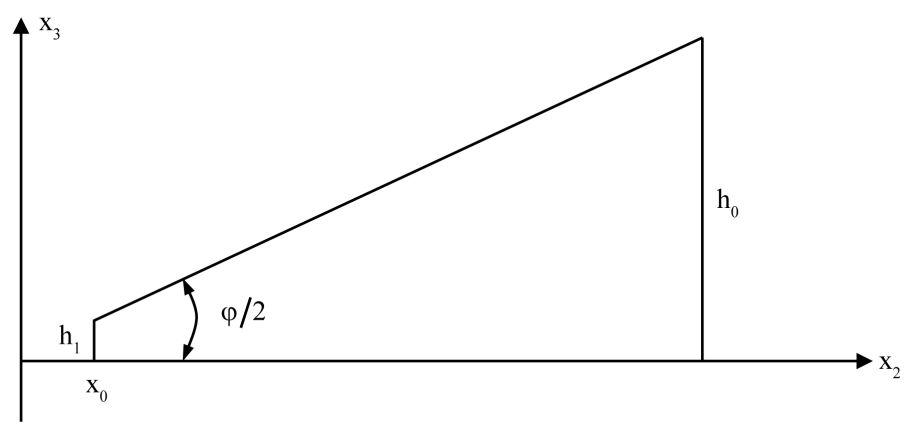

Figure 1. The calculation scheme of plates of variable thickness. 
Here $\Gamma_{E}^{C}\left(\omega_{R}\right)=\int_{x}^{\infty} R(\tau) \cos \omega_{R} \tau \mathrm{d} \tau, \Gamma_{E}^{S}\left(\omega_{R}\right)=\int_{0}^{\infty} R_{\lambda}(\tau) \sin \omega_{R} \tau \mathrm{d} \tau$ respectively, the cosine and sine Fourier transforms of the core material relaxation.

Introducing the notation for the moments $M_{11}=D\left(\frac{\partial^{2} W}{\partial x_{1}^{2}}+v \frac{\partial^{2} W}{\partial x_{2}^{2}}\right), M_{22}=D\left(\frac{\partial^{2} W}{\partial x_{2}^{2}}+v \frac{\partial^{2} W}{\partial x_{1}^{2}}\right)$,

$M_{12}=D(1-v) \frac{\partial^{2} W}{\partial x_{1} \partial x_{2}}, \quad D=\frac{\bar{E} h^{3}}{12\left(1-v^{2}\right)}$ integrating through the strip thickness, will lead Equation (3) her to the following form

$$
\int_{s}\left(M_{11} \frac{\partial^{2} \delta W}{\partial x_{1}^{2}}+2 M_{12} \frac{\partial^{2} \delta W}{\partial x_{1} \partial x_{2}}+M_{22} \frac{\partial^{2} \delta W}{\partial x_{2}^{2}}\right) \mathrm{d} s-\int_{s} \rho h \frac{\partial^{2} W}{\partial t^{2}} \delta W \mathrm{~d} s=0
$$

Integrating twice by parts and equating to zero the coefficients of variations of $\delta \mathrm{W}$ inside the body and on its boundaries, we obtain the following differential equations

$$
\frac{\partial^{2} M_{11}}{\partial x^{2}}+2 \frac{\partial^{2} M_{12}}{\partial x_{1} \partial x_{2}}+\frac{\partial^{2} M_{22}}{\partial x_{2}^{2}}+\rho h \ddot{W}=0, \quad\left(\ddot{w}=\partial^{2} w / \partial t^{2}\right),
$$

with the natural boundary conditions

and the primary alternative to them

$$
\begin{aligned}
& \left\{\begin{array}{l}
\frac{\partial W}{\partial x_{2}}=0 \\
W=0 ; \quad x_{2}=0: l_{2}
\end{array}\right. \\
& \begin{cases}\frac{\partial W}{\partial x_{1}}=0 \\
W=0 ; \quad x_{1}=0: l_{1}\end{cases}
\end{aligned}
$$

$$
\begin{aligned}
& \left\{\begin{array}{l}
M_{22}=0 \\
\frac{\partial M_{22}}{\partial x_{2}}+2 \frac{\partial M_{12}}{\partial x_{1}}=0 ; \quad x_{2}=0: l_{2}
\end{array}\right. \\
& \left\{\begin{array}{l}
M_{11}=0 \\
\frac{\partial M_{11}}{\partial x_{1}}+2 \frac{\partial M_{12}}{\partial x_{2}}=0 ; \quad x_{1}=0: l_{1}
\end{array}\right.
\end{aligned}
$$

Equations (7) and the boundary conditions (8)-(11) are valid for restricted plate. In this case $l_{1} \rightarrow \infty$, instead of (9) and (11) put the radiation conditions [4].

For, constructing of the spectral problem, we introduce the following change of variables

$$
W=W ; \quad \varphi=\frac{\partial W}{\partial x_{2}} ; \quad M=\left(\frac{\partial^{2} W}{\partial x_{1}^{2}}+\frac{\partial^{2} W}{\partial x_{2}^{2}}\right) ; \quad Q=\frac{\partial M_{22}}{\partial x_{2}}+\frac{\partial M_{12}}{\partial x_{1}}
$$

Substituting (12) into (7) we obtain a system of differential equations in partial derivatives that are solved for of the first derivatives respect to $x_{2}$ :

$$
\begin{aligned}
& \frac{\partial Q}{\partial x_{2}}+\frac{\partial^{2} M}{\partial x_{1}^{2}}+D^{\prime}(1-v) \frac{\partial^{2} \varphi}{\partial x_{1}^{2}}+\rho h \frac{\partial^{2} W}{\partial t^{2}}=0 \\
& \frac{\partial M}{\partial x_{2}}-Q-D^{\prime \prime}(1-v) \frac{\partial^{2} W}{\partial x_{1}^{2}}=0 ; \\
& \frac{\partial \varphi}{\partial x_{2}}-\frac{1}{D} M+\frac{\partial^{2} W}{\partial x_{1}^{2}}=0 ; \quad \frac{\partial W}{\partial x_{2}}-\varphi=0 .
\end{aligned}
$$


and alternate boundary conditions on $x_{2}=0: x_{2}=l_{2}: \varphi=0$ or

$$
\begin{gathered}
M-D(1-v) \frac{\partial^{2} M}{\partial x_{1}^{2}}=0 \\
W=0 \text { or } Q+D(1-v) \frac{\partial^{2} \varphi}{\partial x_{1}^{2}}=0 \text { and } x_{1}=0, x_{1}=l_{1}, \varphi=0 \text { or } M-D(1-v) \frac{\partial^{2} M}{\partial x_{1}^{2}}=0, W=0 \text { or } \\
Q+D(1-v) \frac{\partial^{2} \varphi}{\partial x_{1}^{2}}=0
\end{gathered}
$$

We now consider the infinite along the axis $x_{1}$ strip with an arbitrary thickness change $h=h\left(x_{2}\right)$. We seek a solution of the problem (13)-(15) in the form

$$
(Q, M, \varphi, W)^{\mathrm{T}}=(\bar{Q}, \bar{M}, \bar{\varphi}, \bar{W})^{\mathrm{T}} \mathrm{e}^{i\left(\alpha x_{1}-\omega t\right)}
$$

Describing the are harmonic plane waves propagating along the axis $x_{1}$. Substituting (16) into (13) we obtain first order differential equations, solved with respect to derivatives

$$
\left\{\begin{array}{l}
\bar{Q}^{\prime}-\alpha^{2} \bar{M}-\alpha^{2} D(1-v) \bar{\varphi}-\rho h \omega^{2} \bar{W}=0 \\
\bar{M}^{\prime}-\bar{Q}+\alpha^{2} D^{\prime}(1-v) \bar{W}=0 \\
\bar{\varphi}^{\prime}-\frac{1}{D} \bar{M}-\alpha^{2} \bar{W}=0 \\
\bar{W}^{\prime}-\bar{\varphi}=0
\end{array}\right.
$$

with the boundary conditions at the ends of strips, $x_{2}=0, l_{2}$, one of four types

1) simple support: $\bar{W}=\bar{M}=0$

2) sliding clamp: $\bar{Q}=\bar{\varphi}=0$

3) rigid seal: $\bar{W}=\bar{\varphi}=0$

4) the free edge: $\left\{\begin{array}{l}\bar{M}+\alpha^{2} D(1-v) \bar{W}=0 \\ \bar{Q}-\alpha^{2}(1-v) D \bar{\varphi}=0\end{array}\right.$

Thus, formulated by spectral problems (17) to (21) respect to the parameter $\alpha^{2}$, describing the propagation flat flexural of waves in a waveguide embodied in the form strips with an arbitrary thickness change respect to the coordinate $x_{2}$. It is shown that spectral parameter and $\alpha^{2}$ takes only real values (with $R_{E}=0$ ).

We transform for the system (17) and we have

$$
\bar{Q}^{\prime}=\bar{M}^{\prime \prime}+D^{\prime \prime}(1-v) \alpha^{2} \bar{W}+D^{\prime}(1-v) \alpha^{2} \bar{\varphi} \text {, where } M^{\prime \prime}+D^{\prime \prime}(1-v) \alpha^{2} \bar{W}-\alpha^{2} \bar{M}-\rho h \omega^{2} \bar{W}=0
$$

Furthermore

$$
\bar{W}^{\prime \prime}-\frac{1}{D} \bar{M}-\alpha^{2} \bar{W}=0
$$

Thus, the conversion system is of the form

$$
\left\{\begin{array}{l}
M^{\prime \prime}-\alpha^{2} \bar{M}-\left(\rho h \omega^{2}-D^{\prime \prime}(1-v) \alpha^{2}\right) \bar{W}=0 \\
W^{\prime \prime}-\alpha^{2} \bar{W}-\frac{1}{D} \bar{M}=0
\end{array}\right.
$$

The boundary conditions (18)-(21) in is of the form $\bar{W}, \bar{M}$ of variables:

1) simple support: $\bar{W}=\bar{M}=0$

2) sliding clamp: $\bar{W}^{\prime \prime}=\bar{M}^{\prime}-\alpha^{2} D^{\prime}(1-v) \bar{W}=0$ 
3) rigid seal: $\bar{W}=\bar{W}^{\prime}=0$

4) the free edge: $\bar{M}^{\prime}+\alpha^{2} D(1-v)(\bar{W})^{\prime}=0, \bar{M}^{\prime}-\alpha^{2}(1-v)(D \bar{W})^{\prime}=0$

5) at $x_{2}=0$ or $x_{2}=+e_{2}$

Let $\bar{M}$ and $\bar{W}$ some the Eigen functions of the system (22)-(26), possibly complex value. Multiply the equation system (22) $\hat{M}$ and $\hat{W}$ on the complex conjugate of $\bar{M}$ and $\bar{W}$. The identically converting the first equation, we integrate the equalities obtained respect to $x_{2}$ and construct the following linear combination

$$
\begin{aligned}
& {\left.\left[\bar{M}^{\prime}-\alpha^{2}(1-v)(D \bar{W})^{\prime}\right] \hat{W}\right|_{0} ^{l_{2}}+\left.\left[\hat{M}+\alpha^{2}(1-v) D \hat{W}\right] \overline{W^{\prime}}\right|_{0} ^{l_{2}}-\int_{0}^{l_{2}}\left(\bar{M} \hat{W}^{\prime}+\hat{M} \bar{W}^{\prime}\right) \mathrm{d} x_{2}-\alpha^{2} \int_{0}^{l_{2}}(\bar{W} \hat{M}+\hat{W} \bar{M}) \mathrm{d} x_{2}} \\
& -\int_{0}^{l_{2}} \frac{\bar{M} \hat{M}}{D} \mathrm{~d} x_{2}-\omega^{2} \int_{0}^{l_{2}} \rho h \bar{W} \hat{W} \mathrm{~d} x_{2}-2 \alpha^{2}(1-v) \int_{0}^{e_{2}} D^{\prime \prime} \hat{W} \bar{W} \mathrm{~d} x_{2}+\alpha^{2}(1-v) \int_{0}^{e_{2}} D^{\prime}(\hat{W} \bar{W})^{\prime} \mathrm{d} x_{2}=0 .
\end{aligned}
$$

Easy to see that members of not included of the integrals Equation (27) vanish for of any combination of boundary conditions (23)-(26). It is necessary to notice also that all functions under the integral are valid. Expressing $\alpha^{2}$ from (27) we obtain $\left(R_{E}=0\right)$, which

real number.

$$
\alpha^{2}=\frac{\int_{0}^{l_{2}}\left(\bar{M}^{\prime} \hat{W}^{\prime}+\hat{M}^{\prime} \hat{W}^{\prime}\right) \mathrm{d} x_{2}+\int_{0}^{l_{2}} \frac{\bar{M} \hat{M}}{D} \mathrm{~d} x_{2}+\omega^{2} \int_{0}^{l_{2}} \rho h \bar{W} \hat{W} \mathrm{~d} x_{2}}{\int_{0}^{l_{2}}(\bar{M} \hat{W}+\hat{M} \bar{W}) \mathrm{d} x_{2}-2(1-v) \int_{0}^{l_{2}} D^{\prime \prime} \bar{W} \hat{W}^{\prime} \mathrm{d} x_{2}-(1-v) \int_{0}^{l_{2}} D^{\prime}(\bar{W} \hat{W})^{\prime} \mathrm{d} x_{2}},
$$

Thus, it is shown that the square of the wave number for own continuous strip of variable thickness is valid for any combination of boundary conditions.

\section{The Conjugate Spectral Problem the Biorthogonality Condition}

Obtained spectral problems (17)-(21) is not self-adjoint. Had built for her adjoint problem using Lagrange's formula [8] [9]

$$
\int_{0}^{l} L(U) \cdot V^{*} \mathrm{~d} x=\left.Z\left(U, V^{*}\right)\right|_{0} ^{l}-\int_{0}^{l} L^{*}\left(V^{*}\right) \cdot U \mathrm{~d} x
$$

where $L$ and $L^{*}$ —direct and the adjoint linear differential operators; $U$ and $V^{*}$ —arbitrary decisions corresponding boundary value problems.

In this case,

$$
L=\left[\begin{array}{cccc}
\frac{\partial}{\partial x_{2}} & -\alpha^{2} & -\alpha^{2} D^{\prime}(1-v) & -\rho h \omega^{2} \\
-1 & \frac{\partial}{\partial x_{2}} & 0 & -\alpha^{2} D^{\prime}(1-v) \\
0 & -\frac{1}{D} & \frac{\partial}{\partial x_{2}} & -\alpha^{2} \\
0 & 0 & -1 & \frac{\partial}{\partial x_{2}}
\end{array}\right]
$$

So the left-hand side Equation (29) has the following form

$$
\begin{aligned}
& {\left.\left[\bar{Q} \bar{Q}^{*}+\bar{M}^{*}+\bar{\varphi} \bar{\varphi}^{*}+\bar{W} \bar{W}^{*}\right]\right|_{0} ^{l_{2}}-\int_{0}^{l_{2}}\left[\left(Q^{* \prime}+\bar{M}^{*}\right) \bar{Q}+\left(\bar{M}^{* \prime}+\alpha^{2} \bar{Q}^{*}+\frac{1}{D} \bar{\varphi}^{*}\right) \bar{M}\right.} \\
& \left.+\left(\bar{\varphi}^{* \prime}+\bar{W}^{*}+\alpha^{2} D^{\prime}(1-v) \bar{Q}^{*}\right) \bar{\varphi}+\left(\bar{W}^{* \prime}+\alpha^{2} \bar{\varphi}^{*}-\alpha^{2} D^{\prime}(1-v) \bar{M}^{*}+\rho h \omega^{2} \bar{Q}^{*}\right) \bar{W}\right] \mathrm{d} x_{2}=0
\end{aligned}
$$


Thus the conjugate (17)-(21), the system has the form

$$
\left\{\begin{array}{l}
\bar{Q}^{* \prime}+\bar{M}^{*}=0 \\
\bar{M}^{* \prime}+\alpha^{2} \bar{Q}^{*}+\frac{1}{D} \bar{\varphi}^{*}=0 \\
\bar{\varphi}^{* \prime}+\bar{W}^{*}+\alpha^{2} D^{\prime}(1-v) \bar{Q}^{*}=0 \\
\bar{W}^{* \prime}-\alpha^{2} D^{\prime}(1-v) \bar{M}^{*}+\alpha^{2} \bar{\varphi}^{*}+\rho h w^{2} \bar{Q}^{*}=0
\end{array}\right.
$$

Furthermore, we obtain the conjugate boundary conditions of equality to zero outside the integral terms $\left.Z\left(U, V^{*}\right)\right|_{0} ^{l_{2}}$ in the expression (31):

1) simple support: $\bar{\varphi}^{*}=\bar{Q}^{*}=0, x_{2}=0, l_{2}$

2) sliding clamp: $\bar{W}^{*}=\bar{M}^{*}=0, x_{2}=0, l_{2}$

3) rigid seal: $\bar{M}^{*}=\bar{Q}^{*}=0, x_{2}=0, l_{2}$

4) the free edge: $\left\{\begin{array}{l}\bar{\varphi}^{*}-\alpha^{2} D(1-v) \bar{Q}^{*}=0, \\ \bar{W}^{*}-\alpha^{2} D(1-v) \bar{M}^{*}=0, \quad x_{2}=0, l_{2} .\end{array}\right.$

To receive conditions of biorthogonality solutions use once again Lagrange's formula (29) in the form

$$
\int_{0}^{l}\left[L(U) V^{*}+L^{*}\left(V^{*}\right) U\right] \mathrm{d} x=\left.Z\left(U, V^{*}\right)\right|_{0} ^{l_{2}}
$$

And this formula leads to the consideration the following integral

$$
\begin{aligned}
& \int_{0}^{l_{2}}\left[\bar{Q}_{i}^{\prime} \bar{Q}_{j}^{*}-\alpha_{i}^{2} \bar{M}_{i} \bar{Q}_{j}^{*}-\alpha_{i}^{2} D^{\prime}(1-v) \bar{\varphi}_{i} \bar{Q}_{j}^{*}-\rho h w^{2} \bar{W}_{i} \bar{Q}_{j}^{*}+\bar{M}_{i} \bar{M}_{j}^{*}-\bar{Q}_{i} \bar{M}_{j}^{*}+\alpha_{i}^{2} D^{\prime}(1-v) \bar{W}_{i} \bar{M}_{j}^{*}+\bar{\varphi}_{i}^{\prime} \bar{\varphi}_{j}^{*}\right. \\
& \quad-\frac{1}{D} \bar{M}_{i} \bar{\varphi}_{j}^{*}-\alpha_{i}^{2} \bar{W}_{i} \bar{\varphi}_{j}^{*}++\bar{W}_{i} \bar{W}_{j}^{*}-\bar{\varphi}_{i} \bar{W}_{j}^{*}+\bar{Q}_{j}^{* \prime} \bar{Q}_{i}+\bar{M}_{j}^{*} \bar{Q}_{i}+\bar{M}_{j}^{* \prime} \bar{M}_{i}+\alpha_{j}^{2} \bar{M}_{i} Q_{j}^{*}+\frac{1}{D} \bar{M}_{i} \bar{\varphi}_{j}^{*}+\bar{\varphi}_{i} \bar{\varphi}_{j}^{* \prime} \\
& \left.\quad+\bar{W}_{j}^{*} \bar{\varphi}_{i}+\alpha_{j}^{2} D^{\prime}(1-v) \bar{Q}_{j}^{*} \bar{\varphi}_{i}+\bar{W}_{i} \bar{W}_{j}^{* \prime}+\alpha_{j}^{2} \bar{W}_{i} \bar{\varphi}_{j}^{*}-\alpha_{j}^{2} D^{\prime}(1-v) \bar{W}_{i} \bar{M}_{j}^{*}+\rho h \omega^{2} \bar{Q}_{j}^{*} \bar{W}_{i}\right] \mathrm{d} x_{2}=0,
\end{aligned}
$$

where $\left(\bar{Q}_{i}, \bar{M}_{i}, \bar{\varphi}_{i}, \bar{W}_{i}\right)^{\mathrm{T}}$ own form corresponding to the eigenvalue $\alpha_{i}$ original spectral problem; $\left(\bar{Q}_{i}^{*}, \bar{M}_{i}^{*}, \bar{\varphi}_{i}^{*}, \bar{W}_{i}^{*}\right)^{\mathrm{T}}$ - proper form, corresponding to the eigenvalue $\alpha_{j}$ conjugate. Integrating (38) by parts we obtain

$$
\left(\alpha_{i}^{2}-\alpha_{j}^{2}\right) \int_{0}^{l_{2}}\left[-\bar{M}_{i} \bar{Q}_{j}^{*}-D^{\prime}(1-v) \bar{Q}_{j}^{*} \bar{\varphi}_{i}+D^{\prime}(1-v) \bar{W}_{i} \bar{M}_{j}^{*}-\bar{W}_{i} \varphi_{j}^{*}\right] \mathrm{d} x_{2}+\left[D(1-v) \bar{Q}_{j}^{*} \bar{\varphi}_{i}-D(1-v) \bar{W}_{i} \bar{M}_{j}^{*}\right]_{0}^{l_{2}}=0
$$

Whence we have the condition for $i \neq j$ of biorthogonality forms:

$$
\int_{0}^{l_{2}}\left[\bar{M}_{i}+D^{\prime}(1-v) \bar{\varphi}_{i} \bar{Q}_{j}^{*}+\bar{W}_{i}\left(\bar{\varphi}_{j}^{*}-D^{\prime}(1-v) \bar{M}_{j}^{*}\right)\right] \mathrm{d} x_{2}+\left.D(1-v)\left[\bar{W}_{i} \bar{M}_{j}^{*}-\bar{Q}_{j}^{*} \bar{\varphi}_{i}\right]\right|_{0} ^{l_{2}}=\delta_{i j}
$$

In this case expression $\bar{W}_{i} \bar{M}_{j}^{*}-\bar{Q}_{j}^{*} \bar{\varphi}_{i}$ becomes zero if the boundary is set to any of the conditions (18)-(21) in terms the free edge.

Consider the semi-infinite strip along the $x_{1}$ axis variable cross-section, at the end of which the $\left(x_{1}=0\right)$ are given time-harmonic effects:

$$
w=f_{W}\left(x_{2}\right) \mathrm{e}^{i \omega t}, \quad M_{11}=f_{W}\left(x_{2}\right) \mathrm{e}^{i \omega t}, \quad x_{l}=0
$$

We transform boundary conditions (40) so that they contained only the selected variables by us $w, \varphi, M$ and Q 


$$
\begin{gathered}
W=f_{\omega}\left(x_{2}\right) \mathrm{e}^{i \omega t}, \quad M=\left[f_{M}\left(x_{2}\right)+D(1-v) f_{\omega}^{\prime \prime}\left(x_{2}\right)\right] \mathrm{e}^{i \omega t}, \quad x_{1}=0 \\
\frac{\partial W}{\partial x_{1}}=f_{\varphi}\left(x_{2}\right) \mathrm{e}^{i \omega t}, \quad \frac{\partial M}{\partial x_{1}}=\left[f_{Q}\left(x_{2}\right)-D(1-v) f_{\varphi}^{\prime \prime}\left(x_{2}\right)\right] \mathrm{e}^{i \omega t}, \quad x_{1}=0
\end{gathered}
$$

Suppose that the solution of the stationary problem can be expanded in series in Eigen functions of spectral problems solutions. Solution of stationary of the problem (32)-(36), (40)-(42) will be sought as

$$
\left(\begin{array}{c}
W \\
\varphi \\
M \\
Q
\end{array}\right)=\sum_{k=1}^{N} a_{k}\left(\begin{array}{c}
\bar{W}_{k}\left(x_{2}\right) \\
\bar{\varphi}_{k}\left(x_{2}\right) \\
\bar{M}_{k}\left(x_{2}\right) \\
\bar{Q}_{k}\left(x_{2}\right)
\end{array}\right) \mathrm{e}^{-i\left(\alpha_{k} x_{1}-\omega t\right)}
$$

where $\bar{W}_{k}, \bar{\varphi}_{k}, \bar{Q}_{k}, \bar{M}_{k}$-biorthonormal own shape of the spectral of the problem (32)-(36).

Numerical solution of this and all subsequent of spectral tasks was carried out on the computer software complex based on the method of orthogonal pivotal condensation S. K. Godunov [10], combined with the method of Muller. Results obtained by testing the software complex coincide with the results of analytical calculations with an accuracy of 4-5 sign at frequencies from 0.01 to 100 Here and below the entire analysis is conducted in terms of dimensionless of variables in which the material density $\rho$, half the width of the waveguide $l_{2}$ and moments the elastic modulus $E_{01}$ taken equal to unity. As examples of the viscoelastic material take of three a parametric kernel relaxation $R_{\lambda}(t)=R_{\mu}(t)=A \mathrm{e}^{-\beta t} / t^{1-\alpha}$, possesses weak singularity. And also $A=0.048 ; \beta=$ 0.05; $\alpha=0.1$ (Figure 2). Shows the spectral curves of the lower modes of oscillation of the plate of constant thickness corresponding $n=0,1,2,3,4,5$ for the Poisson's ratio $v=0.25$. Analysis of obtained data shows that the range of applicability of the theory Kirchhoff-Love to a plate of constant thickness limited the low frequency range. For example, for the first mode $(n=0)$, because of the unlimited growth of the phase propagation velocity with increasing frequency, For high frequencies $C_{f} C_{s} \sim \sqrt{\omega}$, the range of application of the theory in $0 \leq \omega \leq 3$. At high of frequencies, where the wavelength is comparable or smaller fashion strip thickness arises is known to a localized strips facets a Rayleigh wave, including velocity smaller the speed $C_{s}$.

But it should to note that in the application of the theory Kirchhoff-Lave platinum constant thickness results in a valid conclusion about the growth of the number of propagating modes with growth of frequency, which is well seen from the obtained of spectral curves in Figure 2 proceed to investigate propagation of flexural of waves in a symmetrical strip Kirchhoff-Lava of variable thickness. Consider first waveguide with the linear law thickness change represented in Figure 1 the edges of which are free. Figure 3 gives the dispersion curves of the first mode, depending on the angle of inclination of the face $\varphi / 2$. The figure shows that, unlike the strips of constant cross section in the case of a wedge-shaped the waveguide with a small angle at the base of the wedge $\alpha$ (curve 3 ), the limit of the phase velocity of propagation mode, and

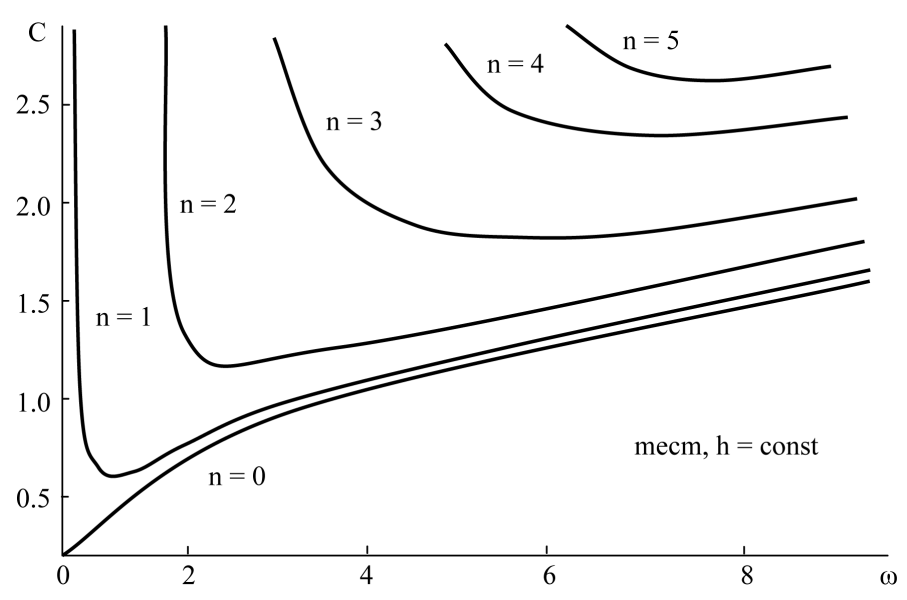

Figure 2. The dependence of the phase velocity on the frequency. 


$$
\lim _{\omega \rightarrow \infty} \tilde{C}_{f}=2 C_{s} \operatorname{tg} \frac{\varphi}{2}
$$

where $C_{5}$-the shear wave velocity, which coincides with the results of other studies [3], and others. Thus, it is shown that the theory of Kirchhoff-Lava allows you to get of waves propagating in a tapered waveguide with a sufficiently small angle at the founding of the wedge velocities less than shear wave velocity and different from the speed of the Rayleigh wave. Furthermore, these waves starting some frequencies distributed without dispersion. Figure 4 shows the mode shapes of the same for the frequency of $\omega=10$, which imply that the band of constant thickness behaves like a rod while in sphenoid strips there is a significant localization of the wave in the zone an acute ciborium, and the larger, the smaller the angle $\varphi$. The above fact gives an explanation of the applicability of the theory Kirchhoff-Lava for studying wave processes in the wedge waveguides, as with growth of frequency with a decreases wavelength parties fashion with another waves is localized with sharp edge of a wedge so that the ratio of the wavelength and the effective thickness of the material is in a the range of applicability of the theory. This assertion is rather, the smaller the angle at the base of the wedge. We can only speak about Numerical stability of results in a large range of variation frequencies, which is confirmed by studies carried out. For example, when $\operatorname{tg} \varphi / 2=0.2$, the values of the phase velocity, the dimensionless the speed to shear wave, at $\omega=3$ and $\omega=50$ differ in the fifth sign which corresponds to calculation precision obtained in the test task. In this example $h_{0}=0.0001$, which of course, gives an increase in the phase velocity as to further increase in frequency, because at such of strong localization of waves to the subtle edge of the wedge are beginning to affect characteristic size - the thickness of the cropped parts of the wedge, and the hypothesis of Kirchhoff-Lava stops working

The numerical experiment showed the absence of the phase velocity of the first mode of the Poisson's ratio

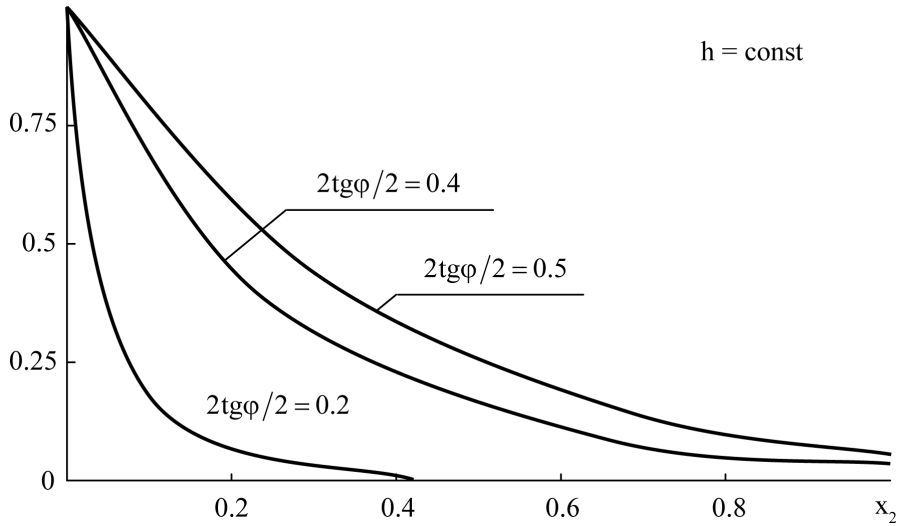

Figure 3. The dependence real and imaginary parts of the phase velocities of the frequency.

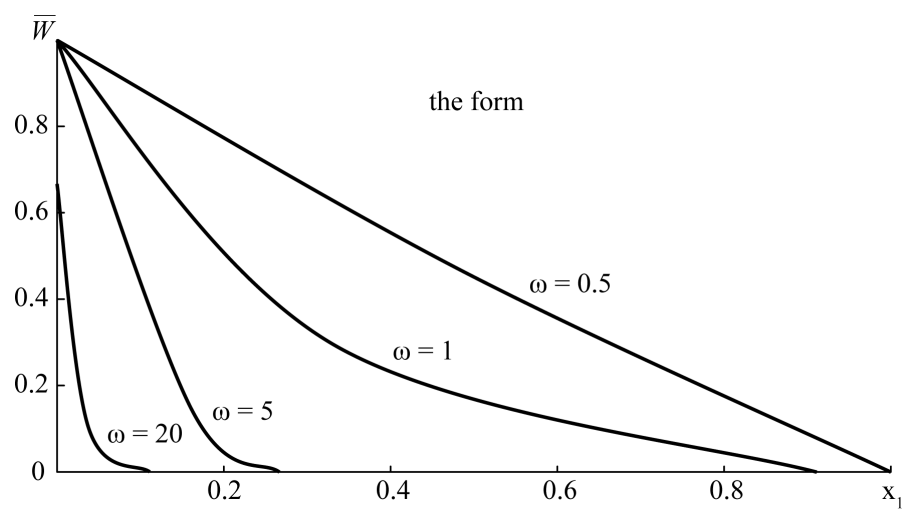

Figure 4. Waveforms along the coordinate $x_{2}$. 
$v$, and also the fact such that the families of dispersion curves with different angles of the wedge have the property of similarity. Attitude of the phase velocity to the limiting does not depend on of the wedge angle $\varphi$. On the modes, starting from the second the limiting velocity dependence on the Poisson ratio becomes noticeably about $9 \%$ for the second mode $0 \leq v \leq 0.5$. In general, the limiting velocity increases with $v$ and the stronger, the greater the number of the mode.

\section{Conclusions}

1) On the basis of variational equations of elasticity theory, we proposed a mathematical statement of the problem of wave propagation in extended plates with variable thickness. We also obtained systems of differential equations and corresponding boundary conditions.

2) It is established that the square of the wave number for endless bands of variable thickness valid for any combination of boundary conditions.

3) In this paper, spectral problem is not self-adjoint, but for her adjoint problem. The conjugate system is composed of ordinary differential equations and the corresponding boundary conditions. With the help of the Lagrange formula, we received biorthogonality conditions of forms. Setting task is solved numerically by the method of orthogonal pivotal condensation S.K. Godunova in combination with the method of Muller.

4) Analysis of obtained data shows that the region with the imaginary the theory Kirchhoff-Love to the plate constant thickness limited the low frequency range. At high frequencies, where the wavelength is comparable to or less than the thickness of the plate, the hypothesis Krihgoffa-Love does not yield reliable results.

5) It is established that, for the phase velocity of propagation modes in the band of variable thickness, there is a finite repartition unlike strip of constant cross section.

\section{References}

[1] Safarov, I.I., Jumayev, Z.F. and Boltayev, Z.I. (2011) Harmonic Wave in an Infinite Cylinder with Radial Cracks with the Damping Material. Journal of Problems in Mechanics, No. 1, 20-25.

[2] Safarov, I.I. and Boltaev, Z.I. (2011) The Propagation of Harmonic of Waves in a Plate of Variable Thickness. Math. Institutions of higher Education, The Povolzhye Region, Series: Phys.-mat. Sciences, No. 4, 31-39.

[3] Safarov, I.I., Teshaev, M.K.H. and Boltaev, Z.I. (2012) Wave Processes in a Mechanical Waveguide. LAP LAMBERT Academic Publishing, Saarbrücken, 217 p.

[4] Grinchenko, V.T. and Myaleshka, V.V. (1981) Harmonic Oscillations and Waves in Elastic Bodies. K.: Science Dumka, 283 p.

[5] Bozorov, M.B., Safarov, I.I. and Shokin, Y.I. (1996) Numerical Simulation of the of Oscillations Homogeneous and Inhomogeneous Dissipative Mechanical Systems. SB RAS, Novosibirsk, 188 p.

[6] Koltunov, M.A. (1976) Creep and the Relaxation. Higher School, Moscow, 276 p.

[7] Sunchaliev, R.M. (1972) Filatov, On Some Methods for Investigation of Nonlinear Tasks of the Theory of Viscoelastic. DAN SSSR, 1972.206, No. 1, 201-203.

[8] Gakhov, F.D. (1963) Boundary Value Problems. Fizmatgiz, Moscow, 639 p.

[9] Neimark, M.A. (1969) Linear Differential Operators. Science, Moscow, 526 p.

[10] Godunov, S.K. (1961) On the Numerical Solution of Boundary Value Problems for Systems of Linear Ordinary Differential Equations. The Success of Mathematical Sciences, 16, 171-174. 